\title{
THE DERIVATIVE OF A MEROMORPHIC FUNCTION
}

\section{J. CLUNIE}

1. It has been shown by Valiron [2] and Whittaker [3] that the derivative of a meromorphic function of finite order is of the same order as the function itself. This result, as pointed out by Whittaker, is equivalent to the following.

THEOREM. If $f(z)$ and $g(z)$ are two integral functions of orders $\rho_{1}$ and $\rho_{2}$ with $\rho_{1}>\rho_{2}$, then $f^{\prime}(z) g(z)-f(z) g^{\prime}(z)$ is of order $\rho_{1}$.

The proofs given by Valiron and Whittaker depend on meromorphic function theory, but in this paper I shall give a proof of the above theorem which depends entirely on integral function theory.

2. A number of lemmas are required and no proof will be given for the first of these as it is already well known.

LEMMA 1 [1, p. 102]. Except for an exceptional set of intervals within which the variation of $\log r$ is finite

$$
z f^{\prime}(z)=N f(z)\{1+o(1)\}, \quad|z|=r,
$$

where $|f(z)|=M(r, f)$ and $N=N(r, f)$.

LEMMA 2. There is an infinite sequence $\left\{\lambda_{i}\right\}_{1}^{\infty}$ such that if $\lambda_{i} \leqq r \leqq \lambda_{i}^{\alpha}$, $\alpha=\left(\rho_{1}-\epsilon / 2\right)\left(\rho_{1}-\epsilon\right)^{-1}$, then

$$
\log N(r, f) \geqq\left(\rho_{\mathbf{1}}-\epsilon\right) \log r .
$$

From the result $[1$, p. 33]

$$
\limsup _{r \rightarrow \infty}\{\log N(r, f)\} / \log r_{i}=\rho_{1}
$$

it follows that there is an infinite sequence $\left\{\lambda_{i}\right\}$ such that

$$
\left\{\log N\left(\lambda_{i}, f\right)\right\} / \log \lambda_{i} \geqq \rho_{1}-\epsilon / 2 .
$$

Since $N(r, f)$ is a nondecreasing function of $r$ this means that

$$
\{\log N(r, f)\} / \log r \geqq \rho_{1}-\epsilon
$$

provided $\left(\rho_{1}-\epsilon / 2\right) \log \lambda_{i} \geqq\left(\rho_{1}-\epsilon\right) \log r$, which gives the lemma.

Received by the editors April 5, 1955. 
LEMма 3. Except when $r$ lies in a set of intervals of total finite length

$$
\left|f^{\prime}(z) / f(z)\right|<r^{p 1+\epsilon}, \quad|z|=r, \epsilon>0 .
$$

If $p$ is the genus of $f(z)$ then

$$
f^{\prime}(z) / f(z)=\rho(z)+\sum_{1}^{\infty} z^{p}\left\{\left(z-a_{n}\right) a_{n}^{p}\right\}^{-1}
$$

where $\left\{a_{n}\right\}$ is the sequence of zeros of $f(z)$ and $\rho(z)$ is a polynomial whose degree does not exceed $\rho_{1}-1$. Hence

$$
\left|f^{\prime}(z) / f(z)\right| \leqq O\left(r^{p^{1}-1}\right)+r^{p} \sum_{1}^{\infty}\left\{\left|r-r_{n}\right| r_{n}^{p}\right\}^{-1} .
$$

Given $\sigma$ and $k>1$ we suppose $\sigma k^{-1 / 2} \leqq r \leqq \sigma k^{1 / 2}$ and consider the above sum in three parts $\sum_{1}, \sum_{2}, \sum_{3}$ such that $r_{n}<\sigma k^{-1}, \sigma k^{-1} \leqq r_{n} \leqq \sigma k$, $r_{n}>\sigma k$ respectively. Using the fact that $p+1$ exceeds the exponent of convergence of the zeros, the sums $\sum_{1}$ and $\sum_{3}$ can be shown to satisfy

$$
\sum_{1}=O\left(r^{p}\right), \quad \sum_{3}=O\left(r^{p}\right)
$$

Integrating $\sum_{2}$ with respect to $r$ over $\left(\sigma k^{-1 / 2}, \sigma k^{1 / 2}\right)$, excluding intervals of length $2 \eta$ centered on each $r_{n}$, where $\eta=\left(\sigma k^{-1 / 2}\right)^{-k}, h>\rho_{1}$, we get

$$
\int \sum_{2} d r=O\left(\sigma^{\rho_{1}+\delta} \log \sigma\right)
$$

provided, given $\delta>0$, we choose $\sigma$ sufficiently large. The range of integration is of length not less than $\left(k^{1 / 2}-k^{-1 / 2}\right) \sigma-2 n(k \sigma)\left(\sigma k^{-1 / 2}\right)^{-h}$, which is $\left(k^{1 / 2}-k^{-1 / 2}\right) \sigma-O(1)$. This means that $\sum_{2}<r^{p_{1}+2 \delta}$ except in a set of intervals whose lengths in sum do not exceed $O\left(\sigma^{-\delta} \log \sigma\right)$. Combining these results gives the lemma.

3. The proof of the theorem will now be given. From the lemmas it follows that we can find an infinite sequence of $r$ such that, with $|z|=r$,

$\log N(\sigma, f) \geqq\left(\rho_{1}-\epsilon\right) \log \sigma, \quad r^{\alpha} \leqq \sigma \leqq r, \quad \alpha=\left(\rho_{1}-\epsilon\right)\left(\rho_{1}-\epsilon / 2\right)^{-1}$,

$$
\begin{gathered}
z f^{\prime}(z) / f(z)=N(r, f)\{1+o(1)\}, \quad|f(z)|=M(r, f), \\
\left|g^{\prime}(z) / g(z)\right|<r p z+e .
\end{gathered}
$$

Also $[1$, p. 32] 


$$
\begin{aligned}
\log M(r, f) & \sim \log \mu(r, f) \\
& =K+\int_{1}^{r} x^{-1} N(x, f) d x \\
& >\int_{r \alpha}^{r} x^{-1} N(x, f) d x \\
& >r^{p_{1}-\epsilon}
\end{aligned}
$$

if $r$ is large enough. Finally

$$
\begin{aligned}
\left|f^{\prime}(z) g(z)-f(z) g^{\prime}(z)\right| & >|f(z) g(z)|\left\{\left|f^{\prime}(z) / f(z)\right|-\left|g^{\prime}(z) / g(z)\right|\right\} \\
& >\exp \left(r^{\rho_{1}-\epsilon}-r^{\rho_{2}+\epsilon}\right)\left(\boldsymbol{r}^{\rho_{1}-1-\epsilon}-r^{\rho_{2}+\epsilon}\right)
\end{aligned}
$$

where use has been made of a result of Borel [1, p. 57]. Thus the theorem follows if $\rho_{1}-1>\rho_{2}$. When this is not the case we choose an integer $n$ so that $n \rho_{1}-1>n \rho_{2}$ and define $F(z)=f\left(z^{n}\right), G(z)=g\left(z^{n}\right)$. Then

$$
F^{\prime}(z) G(z)-F(z) G^{\prime}(z)=n z^{n-1}\left\{f\left(z^{n}\right) g\left(z^{n}\right)-f\left(z^{n}\right) g^{\prime}\left(z^{n}\right)\right\}
$$

and by the previous result $F^{\prime}(z) G(z)-F(z) G^{\prime}(z)$ is of order $n \rho_{1}$. Hence $f^{\prime}(z) g(z)-f(z) g^{\prime}(z)$ is of order $\rho_{1}$ which proves the theorem in this case also.

4. As pointed out by Whittaker the above result is the best possible, as can be seen by taking $f(z)=\cos z$ and $g(z)=\sin z$. However, it would be of interest to know if the theorem was true for functions of the same order if the type of $g(z)$ were less than that of $f(z)$.

It would also be of interest to know what can be said about the order of $f^{\prime}(z) g(z)+f(z) h(z)$ when $g(z)$ and $h(z)$ have orders less than that of $f(z)$.

\section{REFERENCES}

1. G. Valiron, Lectures on the general theory of integral functions, Toulouse, 1923.

2. - Acta Math. vol. 47 (1925) p. 129.

3. J. M. Whittaker, J. London Math. Soc. vol. 2 (1936) pp. 82-87.

University College of North Staffordshire 